VAWKUM Transactions on Computer Sciences

http://vfast.org/index.php/VTCS@2015 ISSN: 2308-8168

Volume 8, Number 1, September-October, 2015

pp-07-19

\title{
THE DESIGN OF NEW LEARNING AUTOMATA FOR PROBLEM GRAPH COLORING
}

\author{
SUSAN KHORRAMDEL*1, HOMAYOUN MOTAMENI ${ }^{2}$ AND FARHAD \\ RAMEZANI $^{3}$ \\ ${ }^{1}$ Babol University Computer Science Graduate Student Tabari, Iran, \\ susan_khorramdel@yahoo.com \\ ${ }^{2}$ Faculty Member, Tabari Institute of Higher Education, Babol, Iran, \\ h_motameni@yahoo.com \\ ${ }^{3}$ Department of Computer Engineering, Islamic Azad University of Sari, Iran, \\ ramezani.farhad@iausari.ac.ir
}

\begin{abstract}
Graph coloring issue, is one of satisfied existing constraints issues in the literature of artificial intelligence. Coloration apical includes assigning color to node graph so that any two adjacent vertices are isochromatic. The minimum number (colors numbers) that we assign to these graphs for coloring are called number of color. This issue is from the group of very difficult issue, NP - complete. Given the importance of graph coloring issue and its many uses, many algorithms suggested finding allowed coloration in graph. Among these can be noted in, exact algorithms, distributed algorithms, parallel algorithms, approximation algorithms and heuristic algorithms, ...The concept of learning Automata at first was introduced by Tstlyn. He was interested in modeling the behavior of biological systems, and definite automata worked in a random environment, introduced as a model for learning. The aim of this research is to present new algorithm on the basis of learning automata to color with accuracy and high speed and the ability to learn graph vertices. The proposed method also has transfer chart and individual performance and this method was examined on the graph with low vertices and high vertices and medium vertices, on the bottom, a number of works steps and total dyes used for coloring of specific graph with optimization algorithms were matched. Evaluation results show high accuracy, speed and its performance of the proposed method is superior to other optimization methods.

Keywords: Learning Automata; Reward; Fine; Graph Coloring; Transfer Diagram.

Abbreviation: $\mathrm{LA}=$ Learning Automata; $\mathrm{GCP}=$ Graph Coloring Problem; $\mathrm{GA}=$ Genetic Algorithms; PGAs = Parallel Genetic Algorithms; MVCP $=$ Minimum Vertex Cover Problem.
\end{abstract}

1. Introduction. In graph theory, coloration graph is one of the special cases, is the issue of labeling of graphs, its general procedures are to use analogous colors to vertices, which this coloration observed special restriction. In the simplest case, a coloration that is desired, in which any two vertices are adjacent (coloration of vertices) which in this case one answer is plausible and to reach to this aim there are various algorithms [1].

The mathematical definition of a graph in order pairs $G=(V, E)$ where $V$ is total of vertices and $E-$ [v] is edge set of graph $\mathrm{G}$ which has $\mathrm{n}$ vertex and m edge, and we indicated maximum degree of vertices with $\Delta[5]$. Minimum of number (numbers of colors) which we assign to this graph for coloring is called color number of edges and show with x́ (G) [2].

The graph coloring problem can be equivalent to a map with the least number of dyes, as any two adjacent cities in the map are not being equal color. Since it can be considered map geography like an equivalent graph, in the special cases, it can be extended to existing methods in graph theory to it, yet many existing methods in artificial intelligence are used to solve this problem [3]. 
2. Prior Work. The practical applications of graph coloring problems include, but are not limited to:

1. Map coloring [4]

2. Scheduling [3]

3. Radio frequency assignment [5]

4. Register allocation [6]

5. Pattern matching

6. Sudoku

In related articles demonstrate the use of genetic algorithms in solving the graph-coloring problem while strictly adhering to the usage of no more than the number of colors equal to the chromatic index to color the various test graphs.

A great deal of research has been done to tackle the theoretical aspects of the graph coloring problem in terms of its generalization as a constraint satisfaction problem [7]. The problem's various applications and solutions have been discussed in detail in porumbel's paper [8].

Evolutionary computation and parameter control has been detailed in a number of papers including ones by Back, Hammel, and Schwefel [9] as well as work by Eiben, Hinterding and Michalewicz [10]. Srinivas and Patnaik examined crossover and mutation probabilities for optimizing genetic algorithm performance [11]. Genetic algorithms and evolutionary approaches have been used extensively in solutions for the graph coloring problem and its applications $[9,12]$.

The concept of utilizing a crowd of individuals for solving NP complete problems has also been the topic of various papers. Most notably the Wisdom of Crowds concept has been used in solving the traveling salesman problem as well as the minimum spanning tree problem [13, 10]. There are generally three approaches to solve the graph coloring problems (GCP) [7-8]:

The first one consists in directly minimizing the number of colors by working in the legal colors space of the problem. In the second approach, the number of colors is fixed and no conflict is allowed, thus, some vertices might not be colored. The objective here is to maximize the number of colored vertices [14, 15]. The third approach consists of first choosing a number of colors $\mathrm{K}$, and then iteratively tries to minimize the number of conflicts for the candidate $\mathrm{K}$.

Whenever a solution with zero conflicts has been found, $\mathrm{K}$ is decremented by one and the procedure continues until were each a $\mathrm{K}$ where the number of conflicts cannot be equal to zero. As a result, the last legal $\mathrm{K}$ will be returned as the best solution [5].

GAs is categorized as global search heuristics and is generally able to find good solutions in a reasonable amount of time.

Common application areas are: scheduling problems [2], graph coloring problems [5], traveling salesman problem, optimization problems [6], and network routing problems for circuit-switched networks, financial marketing, bio-informatics and genomics. GAs is easily parallelized algorithms. PGAs) are parallel implementations of GAs that can provide gain in terms of computational performance and scalability. There exist two major kinds of parallelism in genetic algorithms:

In the computation of the fitness functions of individual and in the application of genetic operators (selection, crossover and mutation). An overview of theoretical advances, computing issues, applications and future trends in parallel genetic algorithms can be found in $[12,15]$ A more detailed discussion of parallel genetic algorithms can be found in [8]. A general framework for studying and analyzing PGAs was proposed by Alba and Troya [10, 15]. Recently, GAs can be applied for supervised and unsupervised data mining sessions. For data mining purposes uses population, individuals as elements defined by attributes and values. These elements or individuals represent candidate production rules.

In order to solve the graph with the genetic algorithm need to follow the below steps:

Step1: turn it in to genetic software.

Step2: Then, consider gene for each chromosome with the graph head numbers.

Step3: for each gene specialize a random color.

In the algorithm [16], each vertex of the graph is equipped with a learning automatic that has two actions in the candidate or non-candidate of the corresponding vertex cover set. Due to characteristics of learning automata, algorithm [16] significantly reduces the number of covering vertices of the graph. The algorithm [16] based on learning automata iteratively minimize the candidate vertex cover through the update its action probability. As the algorithm [16] proceeds, a candidate solution nears to optimal solution of the 
minimum vertex cover problem. MVCP is an NP-complete problem $[17,18]$ which has exponential time complexity. Several practical applications of this problem include communication networks, social network [20], computer network and Bioinformatics [21]. MVCP introduced by Gary and Johnson and proved by Karp as NP-complete problem in connected graph in 1972. Therefore it is almost impossible finding polynomial time complexity algorithm for solving it. Thus many researchers have attracted to solve minimum vertex cover problem [22, 23].

In general, proposed solutions for MVCP are classified in two categories as heuristic and approximation algorithms [20]. Although, exact solution as branch and bound technique has been for this problem, the time complexity is very high [24].

Heuristic algorithms for solving problem [16] have received many attractions for obtaining good approximate and near optimal results in reasonable time. For example, a greedy algorithm starts with an empty set, then, at each step, selects the best candidate with maximum degree and continues to reach the stop condition [25]. A popular approximation technique removes vertices of edges that have been chosen randomly from set. In algorithm [16], the obtained result is always lower than twice the optimum solution, although approximate method in [16] does not optimum solution but can be provided near optimal solution [26].

In recent years, several learning automata based algorithms are presented to solve graph problems and successful results have been reported in literatures such as shortest path problem [27], minimum spanning tree problem [28], minimum dominating set problem [29], maximum clique problem [17, 30] and graph coloring problem [31].

In paper [16], learning automata is applied to solve minimum vertex cover problem. In the algorithm [16], a network of learning automata is created corresponding given graph, the collaboration between learning automata and learning mechanism that exist for each automaton.

In article, this problem is solved by simulating the behavior of insect in wheat farms, namely Eurygaster. These insects attack to farms and when one farm is disrupted, they attack to other farms in a manner that all farms will be disrupted. On the other hand, graph coloring is a NP-Hard problem which by using some Heuristic method like Eurygaster can be solved. The evaluation results show that algorithm is suitable to solve graph coloring problem.

Eurygaster algorithm acts as follows:

First several Eurygasters based on the size of the problems partitions are generated and distributed in the space of the first part to find the solution of the problem. It should be mentioned that if the number of eurygasters is not enough to exhaustedly cover all the space of the related partition, can search all the related space by redistributing them and changing their position so that they can cover all the space mentioned. Eurygaster integriceps is an insect pest that predominantly attacks grains, feeding on the leaves, stems and grains, reducing yield and injecting a toxin into the grains which adds a foul smell to the resulting flour, and substantially reduces the baking quality of the dough [32].

If Euragaster assure that for every pair of adjacent vertices different color can be have its suitability is set to 1 . So the suitability of Eurygaster is 1 or $\infty$. We show this suitability by $S(E)$.

In [33], four learning automata-based approximation algorithms are proposed for solving the minimum (vertex) coloring problem.It is shown that by a proper choice of the parameters of the algorithm, the probability of approximating the optimal solution is as close to unity as possible.

The last proposed algorithm is compared with CHECKCOL [13], GLS [9], ILS [5], TPA [6], AMACOL [1], some well-known coloring algorithms and the results show the efficiency of the algorithm [33] in terms of the color set size and running time of algorithm. Graph coloring problem is widely used in real life applications like computer register allocation, air traffic flow management, timetabling, scheduling, frequency assignment, and light wavelengths assignment inoptical networks. The approximation approaches reported in the literatures can be classified as local search approaches, genetic algorithms, fuzzy-based optimizations, evolutionary algorithms, simulated annealing methods, ant colony-based approaches, Markov chain approaches, and neural network approaches.

Four intelligent approximation algorithms based on learning automata for coloring a given graph with the minimum expected number of colors (minimum coloring problem) as defined in [33].

In each algorithm, a network of learning automata isomorphic to the graph is initially formed by assigning a learning automaton to each vertex of the graph. Such a network of learning automata can be described by a duple $\langle\underline{A}, \underline{\alpha}\rangle$ where $A=\left\{A_{1}, A_{2}, \ldots, A_{m}\right\}$ denotes the set of learning automata and $\underline{\alpha}=$ 
$\left\{\alpha_{1}, \alpha_{2}, \ldots, \alpha_{m}\right\}$ denotes the set of actions in which $\alpha_{i}=\left\{\alpha_{i 1}, \alpha_{i 2}, \ldots, \alpha_{i r i}\right\}$ defines the set of actions can be taken by learning automaton $\mathrm{A}_{\mathrm{i}}$, for each $\alpha_{\mathrm{i}} \in \underline{\alpha}$. The set of colors with which each vertex $\mathrm{V}_{\mathrm{i}}$ can be colored form the set of actions can be taken by learning automaton $\mathrm{A}_{\mathrm{i}}$.

Each of the proposed algorithms contains of a number of stages, and at each stage, each learning automaton randomly selects one of its colors and assigns it to its corresponding vertex. Thus, a coloring is made at each stage. The set of colors to be selected at each stage is referred to as color-set. Then, the algorithm decides whether the coloring is a legal coloring or not (except algorithm 4).

At each stage k, the cardinality of the minimum color-set that has yet been found is denoted by dynamic threshold $T_{K}$. The cardinality of the color-set of a given legal coloring is then compared with dynamic threshold $\mathrm{T}_{\mathrm{K}}$. If it colors the graph with the smaller number of colors, then it is rewarded by the environment, and penalized otherwise. The various legal colorings are iteratively produced and the action probability vectors are updated until a near optimal solution is found to solve the minimum coloring problem with a probability close enough to unity.

In the first proposed algorithm in [33], which call algorithm 1, it is assumed that the number of colors can be assigned to each vertex $\mathrm{Vi}$ is equal to the number of vertices in the graph (i.e., $\mathrm{n}$ ), and therefore the action set of each learning automaton Ai which is referred to as $\alpha_{i}$, also contains the same number of actions. Another method is: Imperialist competitive algorithm (ICA) is a meta-heuristic optimization and stochastic search strategy which is inspired from socio-political phenomenon of imperialistic competition. Imperialist competitive algorithm (ICA) is a meta-heuristic optimization and stochastic search strategy which is inspired from socio-political phenomenon of imperialistic competition [34]. The ICA has excellent capabilities such as high convergence rate and better global optimum achievement. Which, a discrete version of ICA is proposed to deal with the solution of GCP? DICA can produce optimal and valid solutions for different GCP instances.

The GCP is one of the most important classical combinatorial optimization problems. So far, many researchers have been proposed different methods for solving the GCP. These methods fall into some broad categories such as polynomial-time approximation schemes, exact algorithms, greedy methods, parallel and distributed algorithms, decentralized algorithms, and heuristics [35].

One of the most well-known methods in approximation schemes is the successive augmentation [35]. This method assigns a partial coloring to a small number of vertices and this process is extended vertex by vertex until the whole of graph is colored.

Algorithms for finding optimal colorings are frequently based on implicit enumeration [35]. Bruteforce search technique is one of the best well-known exact coloring methods. In these techniques all solutions are checked for finding a reliable and optimal coloring for a graph and have high runtime.

In the greedy algorithms, vertices of the graph are colored in a specific order. The two best examples of greedy algorithms are DSATUR and RLF ${ }^{1}$.

NP complete problems can easily be solved by using distributed computing and parallelism. In the distributed algorithms, graph coloring problem is related to the symmetry breaking problem. Randomized algorithms are faster methods for large dimensional graphs. The fastest algorithm in the class of randomized algorithms is the method presented by Schneider et al.

Since graph coloring problem is an NP-hard problem, several artificial intelligence techniques have been applied on graph coloring problem including algorithms based on neural networks [36], DNA parallel approach (e.g. in [37]), learning automata (e.g. in [38]), evolutionary algorithms, hybrid methods (e.g. in [39] and [40]), scatter search [20], and local search algorithms (e.g. Tabu search [41] or simulated annealing [42]). And et al. presented an approach to the GCP using PSO algorithm that improves a simple deterministic greedy algorithm [43].They proved that their proposed method is better than known heuristic algorithms. Lixia and Zhanli proposed a novel bi-objective genetic algorithm which employs an effective crossover and simple mutation as the genetic operators [44]. The authors claimed that their method is a promising approach to the GCP.

Authors in [45] presented a hybrid chaotic ant swarm approach for the GCP which is called CASCOL. This approach is based on a chaotic ant swarm ${ }^{2}$ and a simple greedy sequential coloring, first-fit algorithm.

1- Recursive Largest First

2- CAS 
In [46] present a hybrid technique that applies a genetic algorithm followed by wisdom of artificial crowds approach to solving the graph-coloring problem. The genetic algorithm described here utilizes more than one parent selection and mutation methods depending on thestate of fitness of its best solution. This results in shifting the solution to the global optimum more quickly than using a single parent selection or mutation method.

Genetic algorithms share an overall structure and workflow yet they vary in the specific details according to the particular problem [47]. The algorithm consists of a parent selection method, a crossover method and a mutation method. The goal algorithm compare with previous algorithm is to improve the fitness of the population by mating its fittest individuals to produce superior offspring that offer a better solution to the problem.

3.Learning Automata. Interests in learning automata began with the outstanding work of Tsetlin in the early in 1960s in the soviet union. Eden brings the automaton theory used by Tsetlin in the field of learning. During last two decades, "Learning Automaton" term has been consistently used by researchers.

Two effective and important factors on automata efficacy are primary conditions and the possibility of fines set by the environment. Primary conditions can be the initial probability vector or condition. One Automata, entirely based on luck is an automata in which any action performed with equal probability. Therefore, an automata that is learning, should have better efficacy that this automata [2].

Learning is defined as changes in performance of a system based on past experiences. One of important features of learning system is the ability to improve their performance in the past. Mathematically, it can be stated that the purpose of a learning system is optimization of a task which is not entirely known [4]. Therefore, one procedure in this issue is reducing of purposes of learning system in an optimization issue that is defined on asset of parameters and its purpose is to find optimal set of parameters [2].

Automata, is a single system which is defined as quintuplet $[\varphi, \mathrm{a}, \beta, \mathrm{F}(0.0), \mathrm{H}(0.0)]$ where $\varphi$ is total internal states. $\alpha$ is output set, $\beta$ is input set, $\mathrm{F}(0.0): \varphi \times \beta \rightarrow \varphi$ is Function by state and current input and $\mathrm{H}$ (0.0): $\varphi \times \beta \rightarrow$ a is automata output function by state and current input.

If the output automata depend only on the current state, it is said to be output state automata. In this case function $H(0.0)$ is converted to $G(0): \varphi \rightarrow$ a [2]. Figurelindicates general shape of learning automata and figure 2 is about feedback connection of automaton and Environment.

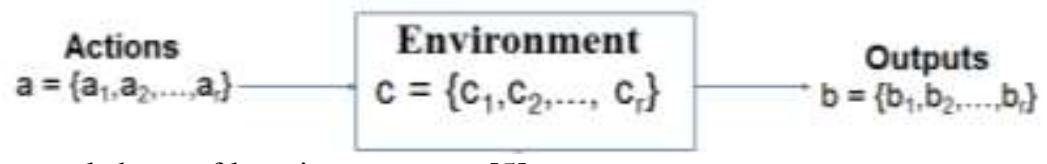

Figure-1: Indicates general shape of learning automata [5].

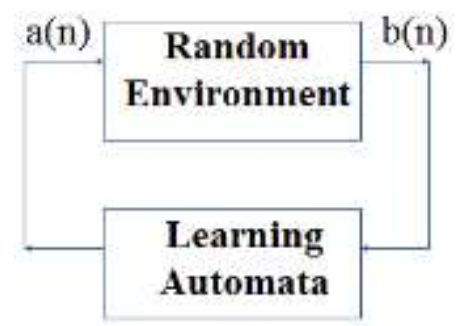

Figure-2: Feedback Connection of Automaton and Environment.

3.1. Definition. A learning automaton is an adaptive decision-making unit situated in a random environment that learns the optimal action through repeated interactions with its environment. The actions are chosen according to a specific probability distribution which is updated based on the environmental response the automaton obtains by performing a particular action.

Briefly, An automaton which selects on of actions related to its past experiences and rewards or punishments from the environment.

3.2. Learning. Ability of a system to improve its responses based on past experience. 
3.3. Environment. The aggregate of all the external conditions and influences affecting the life and development of an organism. The environment can be defined by triple: $\{a, c, b\}$

$\mathrm{a}=\left\{\mathrm{a}_{1}, \mathrm{a}_{2}, \ldots, \mathrm{a}_{\mathrm{r}}\right\}:$ Set of inputs

$b=\left\{b_{1}, b_{2}, \ldots, b_{r}\right\}:$ Set of outputs

$\mathrm{c}=\left\{\mathrm{c}_{1}, \mathrm{c}_{2}, \ldots, \mathrm{c}_{\mathrm{r}}\right\}:$ Set of penalty probabilities

3.4. Environment Models. P-Model: The output can take only two values, 0 or 1

Q-Model: Finite output set with more than two values, between 0 and 1

S-Model: The output is a continuous random variablein the range $[0,1]$

\subsection{Applications.}

$>$ Network routing

$>$ Priority assignment in queuing system

$>$ Task scheduling

$>$ Multiple-access networks

$>$ Image compression

$>$ Pattern classification

4. The proposed method. For the number of nodes in a graph. Received we have learning automata that are colored at the start of coloration by all nodes with one color, which this work is done randomly. So at first total color= 1 and during continuous process, adjacent vertices which have same color should change color the minimum of colors may be allocated to it. So in this regard we need to separate neighboring vertices of non - neighboring vertices.

During coloration, if a vertex has similar color to its neighboring vertices, vertex reviewed is fine, which this fine has 2 modes that in these cases, we fully express them.

5 times, change of colors takes place, and if it has also similar color, we should apply a new color, but if it has different color with its neighboring vertex, will reward and vertices color remains constant.

At the end of algorithm, to ensure, we check resulting coloration again, whether or not it has arrived to right answer.

That from auxiliary variable flag we have used to check, as long as acceptable coloration returns as result, algorithm trend is repeated and with color changes of nodes by increasing of colors (in the minimized mode) we attempt to consider vertex colors so that any neighboring vertices are iso chromatic.

As explained, an algorithm is composed of an infinite loop and as long as it is running that an acceptable coloration leads as results and results of each repeat namely, the number of colors allocated to graph keep in any step.

The input graph is defined as an array. The columns of this array indicate the number of vertices, so if we have $\mathrm{n}$ vertices, array should have $\mathrm{n}$ column of int.

This array is composed of 3 lines:

First line: includes number of colors is called color No.

Second line: includes automata memory is called memory No.

Third line: includes numbers of color with are colored wrong during coloration running and caused to fine is called wrong color.

4.1. Transfer chart. Recommended automata named $\mathrm{kh}_{3,5}$ that transfer chart or its status change is as figure 


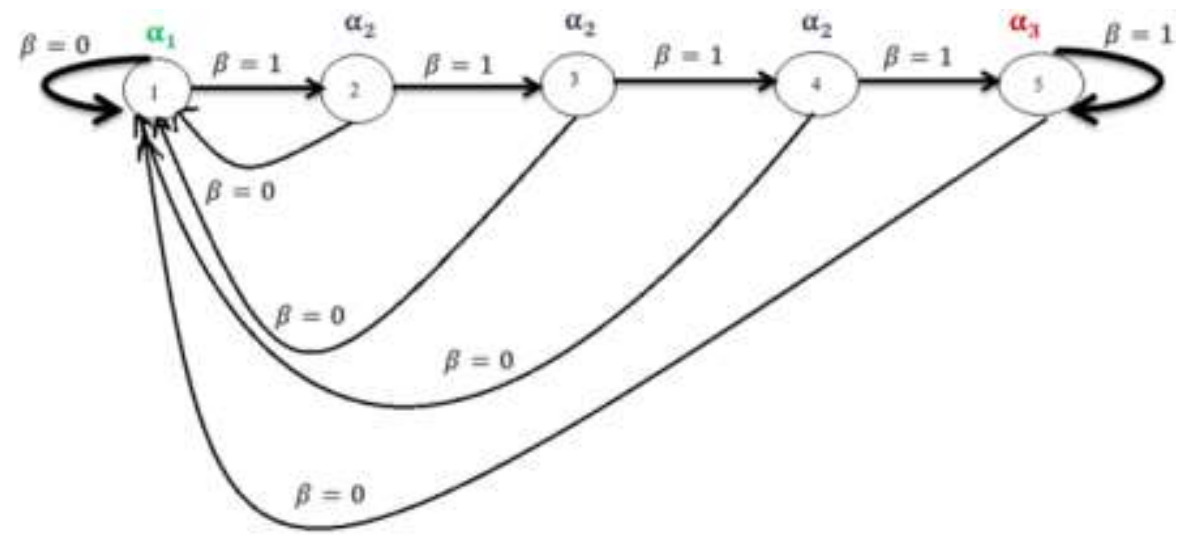

Figure-3: Transfer chart $\mathrm{kh}_{3,5}$.

This linear automata has 5 mode which are numbered 105 , and also we have 3 actions.

$\mathrm{a}_{1}=$ constant color

$\mathrm{a}_{2}=$ change color

$\mathrm{a}_{3}=$ apply the new color

Similar to another automata we have 2 Rewards $\beta=0, \beta=1$ which are considered for Reward and penalty respectively. If a vertex has equal color with its neighboring vertex, it should be fine that is $\beta=1$ and in contrast, if a vertex has different color with its neighboring vertex, it should get rewards, $\beta=0$ for this vertex.

4.2. Reward Action. This process composed of following steps:

Step (1) checks the adjacent vertices:

In this step at first it should be studied whether or not vertices are neighboring, which if it is true, we authorized to go to the next step.

Step (2) checks the color of adjacent vertices:

To study the color of vertices, first line of corresponding array to its vertices should be checked. If neighboring vertices have different color, checking vertex should rewarded otherwise, should be fined.

Step 3: Reward of neighboring vortices:

When 2 neighboring vertices have different color, should be rewarded and the color of that vertex should remain unchanged and memory no value (third line of array) vertex become equal to zero.

In summary in the chart of status change we came back to the first mode with receiving reward from each state and the color of vertices remain constant.

4.3. Punishments Action. Penalty act in general occurred in 2 different times.

1) Memory number value lower or equal to 5 that the color of node with other vertices finds color change randomly, and added a unit to memory No value.

2) If memory No value become larger than 5 , in fact, change of new color is allocated and memory no value changes to zero.

4.3.1. Change the color of the nodes. If Memory No value was $\leq 5$, we should add an unite to memory no value, and other vertices color except 2 reviewed vertices are considered for that vertex randomly and change of color is done.

4.3.2. Apply the new color. In this step, we should do 2 sequential works as follow:

First step: The Memory No value of a node we make it zero.

Second step: In this step it should be allocated a new color to this vertex, that how to add new color is that at first we should get largest assigned color that is numerical value of last variable of Total color and then we add one unite to this value and resulting number is assigned as new color to the node. 
5. Simulated results. The algorithms are implemented using MATLAB software; our test was run on desktop computer with following specifications.

CPU: Intel Core (TM) i3 CPU M350 @ 2.27GHz2.27GHz

RAM: $4.00 \mathrm{~GB}$ (3.23 GB usable)

OS: windows 7

Data used to test our approach are derived from the DIMACS benchmarking graph collection. DIMACS is the center for discrete mathematics and Theoretical Computer Science. It is part of Rutgers university (the state university of New Jersey Rutgers et al. 2011).The data are frequently used in challenges involving constraint satisfaction problems.

for evaluation algorithm Suppose have a set of colors $C=\left\{c_{1}, c_{2}, \ldots, c_{n}\right\}$ and a set of vertices $V=\left\{v_{1}, v_{2}, \ldots\right.$, $\left.\mathrm{v}_{\mathrm{n}}\right\}$ and a set of edges $\mathrm{E}=\left\{\mathrm{e}_{1}, \mathrm{e}_{2}, \ldots, \mathrm{e}_{\mathrm{n}}\right\}$.

According to partition, give $\mathrm{c}_{1}$ to the first vertex so all of the vertices that are adjacent to first vertices can't take c1, but other vertices can have this color. After it, when want to color another vertex, if it is adjacent to first vertex it can't take $c_{1}$; otherwise it take $c_{1}$ to satisfy the minimalistic of used color to assign to vertices. According to this routine, Eurygaster is constituted in each partition.

To evaluate and compare of proposed algorithm with this algorithm, have chosen 5 files from DIMACS benchmarking graph collection. The number of vertices and edges in these files are different.

Table1 shows the used color number and running time of proposed approach against genetic algorithm and Eurygaster algorithm.

Table 1.The evaluation result of proposed approach against genetic algorithm and Eurygaster algorithm

\begin{tabular}{|c|c|c|c|c|c|c|c|c|}
\hline File name & Nodes & Edges & $\begin{array}{l}\text { CN } \\
\text { The number of tured } \\
\text { Color in proposed } \\
\text { appeoach [33] }\end{array}$ & $\begin{array}{l}\mathrm{CN} \\
\text { The number of used } \\
\text { Color in genetic } \\
\text { Algonithm }\end{array}$ & $\begin{array}{l}\text { CN } \\
\text { The number of used } \\
\text { Color in proposed } \\
\text { Approuch }\end{array}$ & $\begin{array}{c}\text { RT(sec) } \\
\text { Running Time } \\
\text { of our } \\
\text { approach } \\
{[33] \text { is second }}\end{array}$ & $\begin{array}{l}\text { RT(ses) } \\
\text { Running } \\
\text { Tume } \\
\text { of Genetic } \\
\text { algovithm } \\
\text { in second }\end{array}$ & $\begin{array}{l}\text { RT(sec) } \\
\text { Rusaning } \\
\text { Time } \\
\text { Of our } \\
\text { approach } \\
\text { in secand }\end{array}$ \\
\hline Myocil3.col & 11 & 20 & 4 & 4 & 4 & 0.002 & 0.007 & 0.002 \\
\hline Myceil4.col & 23 & 71 & 5 & 5 & 5 & 0.007 & 0.021 & 0.003 \\
\hline Quecn6_6.ced & 25 & 290 & 7 & 8 & 6 & 2.120 & 14,043 & 0.217167 \\
\hline Huck.ool & 74 & 301 & 11 & 11 & 8 & 0.011 & 0.559 & 0.013689 \\
\hline David.col & 87 & 406 & 11 & 11 & 9 & 0.128 & 0.540 & 0.021356 \\
\hline
\end{tabular}

As you can see the number of color in proposed approach is optimal while in genetic algorithm and Eurygaster algorithm in some benchmark the number of color is not optimal. Also in proposed approach, running time is less than another algorithm. The reason for this is that in genetic algorithm after passing from some generation, some chromosome may be produced that existed in previous generations. This schema cause's genetic algorithm is rune slowly. The efficiency of the proposed method is compared algorithm [48] with GA on five well-known graph coloring benchmarks. These benchmarks are Dataset1, Dataset2, Myceil3.col, Myceil4.col and queen5_5.col these data sets cover examples of data of low, medium and large dimensions. Table 2 summarizes the characteristics of these benchmarks. Also Table 3 and 4 indicates the parameters set for DICA and GA in our implementations.

Dataset1 is a complete graph which has 15 vertices and 105 edges. The chromatic number of this graph is 15. Dataset2 is another complete graph which has 20 vertices and 190 edges and its chromatic number is 20. Myceil3.col has 11 vertices and 20 edges. Myceil4.col has 23 vertices and 71 edges. The chromatic number for Myceil3.col, Myceil4.col are 4, 5 respectively. Queen5_5.col has 25 vertices and 160 edges and the chromatic number for Queen5-5 is 5.

Another algorithm that compare with proposed algorithm:

Table5 shows the results (over 20 runs) obtained based on this measure. The results show the proposed method often works very well and finds the valid and optimal solution for different GCP instances. 
Table 2. Characteristics of Data Sets considered

\begin{tabular}{|l|l|l|l|l|}
\hline \multicolumn{1}{|c|}{ Graph } & $\begin{array}{c}\text { Number of } \\
\text { Vertices }\end{array}$ & $\begin{array}{c}\text { Number of } \\
\text { Bdges }\end{array}$ & \multicolumn{1}{|c|}{ Chromatic Number } & \multicolumn{1}{c|}{$\begin{array}{c}\text { Chromatic Number } \\
\text { proposed algorithm }\end{array}$} \\
\hline Dataset 1 & 15 & 105 & 15 & 6 \\
\hline Dataset 2 & 20 & 190 & 20 & 6 \\
\hline Myceil3.col & 11 & 20 & 4 & 4 \\
\hline Myceil4.col & 23 & 71 & 5 & 5 \\
\hline Queen5_5.col & 25 & 160 & 5 & 4 \\
\hline
\end{tabular}

Table 3. The DICA algorithm parameters setup

\begin{tabular}{|l|l|}
\hline Parameter & Value \\
\hline Population size & 300 \\
\hline Number of Initial Imperialists & $10 \%$ of population size \\
\hline Number of All Colonies & All population except imperialists \\
\hline Number of Decades iteration count & 100 \\
\hline Revolution Rate & 0.25 \\
\hline Uniting Threshold & 0.02 \\
\hline Assimilation Coefficient & 1.50 \\
\hline Assimilation Angle Coefficient & 0.50 \\
\hline Damp Ratio & 0.90 \\
\hline
\end{tabular}

Table 4. The GA algorithm parameters setup

\begin{tabular}{|l|l|}
\hline Parameter & Value \\
\hline Population size & 300 \\
\hline Mutation rate & 0.25 \\
\hline Selection probability & 0.50 \\
\hline Number of Generation / Iteration count & 100 \\
\hline
\end{tabular}

Table 5. Results of DICA and GA algorithms against proposed algorithm on five data set, the quality of solutions is evaluated using efficiency metric. The table shows success

\begin{tabular}{|c|c|c|c|c|c|}
\hline Gropt & Nimiber of vertices & Nunber of Edpes & D\&CA Success & GA Soxces & Proposed Al porithan Sraccess \\
\hline Drtaket : & 15 & 105 & 30 & 20 & 6 \\
\hline Drtaset 2 & 20 & 190 & 19 & is & 10 \\
\hline Myceibed & 11 & 20 & 20 & 20 & 5 \\
\hline Mycellecol & 23 & $7 \mathrm{t}$ & 20 & 18 & 5 \\
\hline Quecen $5 . \mathrm{cel}$ & 25 & 160 & 18 & 16 & 12 \\
\hline
\end{tabular}

Also simulations show the size of population, the number of initial imperialist countries, the revolution rate, and also use an appropriate strategies for implementing the assimilation and revolution operators (in DICA) are effective to reach the optimal solutions.

As mentioned in above sections, like to the mutation in the GA technique we selected a low revolution rate. For graphs that have few vertices we can use an initial population with fewer individuals and for high dimensional graphs we use a large initial population and also we can increase the number of iterations. In GA method, among different selection methods, we used roulette wheel to choose individuals to create next population. Also 2-pt crossover is used in the recombination process. Selection and mutation rate are $0.5,0.3$ respectively. For DICA the revolution rate and uniting threshold are set to 0.25 and 0.02 
respectively. As shown in Table 2, for Dataset1 the number of successful colorings of DICA and GA are same. For Dataset2 the number of successful iterations of DICA is greater than GA.

The number of successful iterations of DICA for Dataset2, Myceil4.col and queen5-5.col data sets is greater than GA. Simulation results indicate the runtime of DICA is lower than GA over on all data sets and this is due to the high convergence rate of the DICA method But As is observed, present method requires to less colors for coloration of graph and also proposed algorithm approaches to less repeat for optimum solution and has higher rate convergence.

7 files were chosen from the DIMACS collection. The graphs the files represent vary in vertex count, edge count and overall complexity. The vertex count ranges between 11 and 561 and the edge count ranges from 20 to 11654. The rationale behind the selection of these graphs other than the wide range of variation is that there is a known chromatic number for each of them, or at least a good approximation.

The following files were used in this approach:

(myciel3.col, myciel4.col, queen5_5.col, queen6_6.col, huck.col, jean.col, david.col).

Table 6 displays the following statistics for each file:

- The number of vertices $|\mathrm{V}|$

- The number of edges $|\mathrm{E}|$

- The expected chromatic number $\chi(\mathrm{G})$

- The minimum number of colors used by this algorithm $\mathrm{k}_{\min }$

- The minimum number of colors used by a comparative publication using a hybrid parallel genetic algorithm (HPGAGCP)

-Average time it took to find a solution as seen in the above table our proposed method in less time and with less color is the optimal solution.

Table 6.Results of running the Proposed algorithm on 7 .col files from the DIMACS collection

\begin{tabular}{|c|c|c|c|c|c|c|c|c|}
\hline File & V & E & $x(G)$ & $K_{m}$ & HPGAGCP & CX Propesed algonthm & RT(sec) Algarithm [46] & RT(see) Proposed algorthm \\
\hline Myceili.col & 11 & 20 & 4 & 4 & 4 & 4 & 0003 & 0.002256 \\
\hline Myscrilt, col & 23 & 71 & 5 & 5 & 5 & 5 & 0.006 & 0.003782 \\
\hline Queen'5 5. mol & 23 & 160 & 5 & 5 & 5 & 5 & 0.031 & 0.077086 \\
\hline Quecta6 6..col & 25 & 290 & 7 & 7 & 8 & 6 & 6.100 & 0.217167 \\
\hline Hockeal & 74 & 301 & II & II & II & 8 & 0.015 & 0.013689 \\
\hline Jean coll & 50 & 254 & 10 & 10 & 10 & 7 & 0.015 & 0.016351 \\
\hline Davideol & 87 & +06 & 11 & II & 11 & 9 & 0.019 & 0.021396 \\
\hline
\end{tabular}

6. Conclusion. In this article, for coloration of graph from new learning automata and with unique transfer chart and action is used. that the reason of proposed idea is improving of existing weak nesses in the past methods and reach to ultimate goal hence to gain minimum number of required color for coloration of graph vertices and also presents allowable coloration.

By analyzing the results, it can be stated certainly that these results from research simply and also with the best optimality results.

From the advantages of this method can be referred to above:

The ability to apply process for graph with small, medium and large dimensions, To obtain the most efficient possible state, The number of required colors for coloration of graph in each step, The total number of required colors for coloration of graph, high resolution, High rate of convergence and The ability to learn automata unlike other methods.

From disadvantages of this method it can be noted that:

Learning time of less number of nodes with the case of a large number of nodes does not matter.

[1]. Kosar, N., Petrickova, S., \& Reiniger, B. (2014). A note on list-coloring powers of graphs. Discrete Mathematics, 332, 10-14.

[2]. Narendra, K. S., \& Thathachar, M. A. L. (1989). Learning Automata: An Introduction.Prentice Hall.

[3]. Oommen, B. J., \& Ma, D. C. Y. (1988). Deterministic learning automata solution to the keyboard optimization problem. The IEEE Transactions on Computers, 37(1), 2-3. 
[4]. Meybodi, M. R., \& Beigy, H. (1998). New class of learning automata based scheme for adaption of back propagation algorithm parameters. Proceeding of EUFIT-98; 7-10 Sep; Aachen, Germany: pp. 339-344.

[5]. Beigy,H.,\&Meybodi, M. R. (1999) Optimization of topology of neural networks using learning automata. Proceeding of $3^{\text {th }}$ Annual International Computer Society of Iranian Computer Conference, CSICC 98, Tehran, Iran: pp. 417-428.

[6]. D'Adderio, M., \& Moci, L. (2013). Graph colorings flows and arithmetic Tuttepolynomial. The Journal of Combinatorial Theory, 120,11-27.

[7]. Burke, E., \& Newall, J.A. (2007). Multi-stage evolutionary algorithm for the timetable problem. The IEEE Transactions on Evolutionary Computation, 63-74.

[8]. Carter, M., Laporte, G., \& Lee S. Y. (2006). Examination timetabling: algorithmic strategies and applications. Journal of the Operational Research Society, 47, 373-383.

[9]. Hertz, A., \& Werra de, D. (1987). Using tabu search techniques for graph coloring. Computing, 39(4), 345-351.

[10]. Mabrouk, B. B., Hasni, H., \& Mahjoub, Z. (2009). On a parallel genetic-tabu search based algorithm for solving the graph colouring problem. European Journal of Operational Research, 197(3), 11921201.

[11]. Ashby, L. H., Leif, H., Yampolskiy, R. V., \& Roman, V. (2011). Genetic algorithm and wisdom of artificial crowds algorithm applied to light up. In: IEEE $201116^{\text {th }}$ International Conference on Computer Games: AI, Animation, Mobile, Interactive, Multimedia, Educ\& Serious Games; 27-30. Louisville, KY, USA: IEEE. pp. 27-30.

[12]. Chen, J., Antipov, E., Lemieux, B., Cedeno, W., \& Wood D. H. (2008). DNA computing implementing genetic algorithms. In: Landweber LF, Winfree E, Lipton R, Freeland S, editors. Evolution as Computation. New York, Springer Verlag, pp. 39-49.

[13]. Burke, E., \& Petrovic, S. (2002). Recent research directions in automate timetabling. European Journal of Operational Research, 140(2), 66-280.

[14]. Glass, C., \& Prugel-Bennett, A. (2009). Genetic algorithm for graph coloring: Exploration of Galinier and Hao's algorithm. Journal of Combinatorial Optimization, 229-236.

[15]. Konfrst, Z. (2011) Parallel genetic algorithms: Advances. Computing trends, Applications and Perspectives.Proceeding of $18^{\text {th }}$ International Parallel and Distributed Processing Symposium (IPDPS'04).

[16]. Mousavian, A., Rezanian, A. R., \& Meybodi, M. R. (2013). Solving Minimum vertex cover problem using learning automata. In: Iranian Conference; 28 Nov. Iran. pp. 5.

[17]. Masoumi, B., \& Meybodi, M. R. (2011). Speeding up learning automata based multi agent systems using the concepts of stigmergy and entropy. Journal Expert Systems with Applications, 38(7), 81058118 .

[18]. Torkestani, J. A., \& Meybodi, M. R. (2011). Learning automata based algorithms for solving stochastic minimum spanning tree problem. Journal Applied Soft Computing, 11(16), 4064-4077.

[19]. Amiri, F., Yazdani, N., Faili, H., \& Rezvanian, A. (2013). A novel community detection algorithm for privacy preservation in social networks. In: Intelligent Informatics Abraham, Ed, Springer Berlin Heidelberg, pp. 443-450.

[20]. Jovanovic, R., \&Tuba, M. (2011). An ant colony optimization algorithm with improved pheromone correction strategy for the minimum weight vertex cover problem. Application of soft computing, 11, 5360-5366.

[21]. Rezvanian, A., \& Meybodi, M. R. (2010).Tracking extreme in dynamic environments using a learning automata-based immune algorithm.In: Grid, Distributed Computing, Control and Automation, KimT, Ed, Springer Berlin: Heidelberg, pp. 216-225.

[22]. Halperin, E. (2012). Improved approximation algorithms for the vertex cover problem in graphs and hyper graphs. SIAM Journal on Computing, 31, 1608-1623.

[23]. Torkestani, J. A., \& Meybodi, M. R. (2012). Finding minimum weight connected dominating set in stochastic graph based on learning automata. Information Sciences, (200), 57-77.

[24]. Cai, S., Su, K., \& Sattar, A. (2011). Local search with edge weighting and configuration checking heuristics for minimum vertex cover.Artificial Intelligence, 175, 1672-1696. 
[25]. Escoffier, B., Gourves, L., \& Monnot, J. (2010). Complexity and approximation results for the connected vertex cover problem in graphs and hyper graphs.Journal of Discrete Algorithms, 8, 3649.

[26]. Zhang, Z., Gao, X., \& Wu, W. (2009) Algorithms for connected set cover problem and fault-tolerant connected set cover problem. Theoretical Computer Science, 410, 812-817.

[27]. Torkestani, J. A., \& Meybodi, M. R. (2011). A cellular learning automata based algorithm for solving the vertex coloring problem. Expert Systems with Applications, 38(8), 9237-9247.

[28]. Torkestani, J. A., \& Meybodi, M. R. (2011). Learning automata based algorithms for solving stochastic minimum spanning tree problem.Journal of Applied Soft Computing, 11(16), 4064-4077.

[29]. Torkestani, J. A., \& Meybodi, M. R. (2012). Finding minimum weight connected dominating set in stochastic graph based on learning automata. Information Sciences, 200, 57-77.

[30]. Soleimani-Pouri, M., Rezvanian, A., \& Meybodi, M. R. (2012). Finding a maximum clique using ant colony optimization and particle swarm optimization in social networks.In: IEEE Proceedings of the International Conference on Advances in Social Networks Analysis and Mining (ASONAM).Washington, DC, USA: IEEE. pp. 58-61.

[31]. Torkestani, J. A., \& Meybodi, M. R. (2011). Cellular learning automata based algorithm for solving the vertex coloring problem. Expert Systems with Applications, 38(8), 9237-9247.

[32]. Ahmadi, F. (2013). Eurygaster Algorithm: a new approach to optimization. International Journal of Computer Application, 324-329.

[33]. Akbari Torkestani, J., \& Meybodi, M. R. (2009). Graph coloring problem based on learning automata. International Conference on Information Management and Engineering, 2009. ICIME '09. 3-5 April, Kuala Lumpur, pp. 718-722.

[34]. Atashpaz-Gargari, E., \& Lucas, C. (2007). Imperialist competitive algorithm: an algorithm for optimization inspired by imperialistic competition. In: IEEE Congress on Evolutionary Computation, 25-28 September; Singapore: IEEE. pp. 4661-4667.

[35]. Ramani, A., Markov, I. L., Sakallah, K., \& Aloul, F. (2006). Breaking instance-independent symmetries in exact graph colouring. Journal of Artificial Intelligence Research, 289-322.

[36]. Jagota, A. (1996). An adaptive, multiple restarts neural network algorithm for graph coloring. European Journal of Operational Research, 257-270.

[37]. Chung-Wei, Y., \& Kee-Rong, W. (2009). A novel DNA-based parallel computation for solving graph coloring problems. In: WRI World Congress on Software Engineering, WCSE '09. pp. 213217.

[38]. Torkestani, J. A., \& Meybodi, M. R. (2009). Graph coloring problem based on learning automata. In: IEEE International Conference on Information Management and Engineering; 3-5 April; Kuala Lumpur: IEEE. pp. 718-722.

[39]. Galinier, P., \& Hao, J. K. (1999). Hybrid evolutionary algorithms for graph coloring. Journal of Combinatorial Optimization, 3(4), 379-397.

[40]. Fleurent, C., \& Ferland, J. A. (1996). Genetic and hybrid algorithms for graph coloring. Annals of Operations Research, (63), 437-461.

[41]. Hamiez, J. P., \& Hao, J. K. (2002). Scatter search for graph coloring. In: Lecture Notes in Computer Science, 10 April. pp. 168-179.

[42]. Chams, M., Hertza, D., \& Werra, D. (1987) .Some experiments with simulated annealing for coloring graphs. European Journal of Operational Research, 32: 260-266.

[43]. Lixia, H.,\&Zhanli, H. (2010). A novel bi-objective genetic algorithm for the graph coloring problem.In: IEEE Second International Conference on Computer Modeling and Simulation; 22-24 Jan. Sanya, Hainan: IEEE. pp. 3-6.

[44]. Ge, F., Wei, Z., Tian, Y., \& Huang, Z. (2010). Chaotic ant swarm for graph coloring. In: IEEE International Conference on Intelligent Computing and Intelligent Systems (ICIS); 29-31 Oct. Xiamen. pp. 512-516.

[45]. Mohamed, D. S., \& Elbernoussi, S. (2011). Max-min ant system for the sum coloring problem. In: IEEE International Conference on Communications, Computing and Control Applications (CCCA); 3-5 March.Hammamet. pp. 1-4. 
[46]. Hamidi, M., \& Yampolskiy, V. (2012). Genetic algorithm applied to the graph coloring problem.Computer Engineering and Computer Science. J.B. Speed School of Engineering, Louisville, Kentucky, p. 1-7.

[47]. Shby, Leif, H., Yampolskiy, \& Roman, V. (2011). Genetic algorithm and wisdom of artificial crowds algorithm applied to light up. In: IEEE the $16^{\text {th }}$ International Conference on Computer Games: AI, Animation, Mobile, Interactive Multimedia, Educational \& Serious Games; 27-30 July. Louisville, KY, USA: IEEE. 27-30.

[48]. Emami, H., \& Lotfi, S. H. (2013). Graph coloring problem based on discrete imperialist competitive algorithm. International Journal in Foundations of Computer Science \& Technology, (IJFCST), 3(4). 